\title{
Modes in Single-Negative Slab with Tensor Material Parameters
}

\author{
Masashi Hotta $^{1)}$, Mitsuo HANo ${ }^{1)}$, and Ikuo AWAI ${ }^{2)}$ \\ ${ }^{1)}$ Department of Electrical \& Electronic Engineering \\ Graduate School of Science \& Engineering \\ Yamaguchi University \\ 2-16-1 Tokiwadai, Ube 755-8611, JAPAN \\ Phone: +81-836-85-9436, Fax.: +81-836-85-9401 \\ e-mail: hotta@yamaguchi-u.ac.jp \\ 2) Department of Electronics and Informatics, Ryukoku University \\ Seta, Otsu 520-2149, JAPAN
}

\begin{abstract}
Modal analysis of an anisotropic single-negative slab with tensor material parameters are presented. By the comparison with the eigenvalue equation of surface modes along single-negative slab with negative scalar permeability, the validity of the present study is confirmed. We have also examined which elements of the material parameter tensors affect existence of modes in the slab. Giving consideration to the dispersion of material parameters, we demonstrate in detail that TE modes propagate in a slab with one negative element of the permeability tensor numerically. These TE modes turn out to be the magnetostatic waves (MSWs), which is the first demonstration of the MSW in a nonmagnetic material.
\end{abstract}

Index Term - Electrostatic analysis, Magnetostatic surface waves, Magnetostatic volume waves, Metamaterial

\section{INTRODUCTION}

Much study has been carried out on metamaterials with negative material constants after Pendry's implementation of negative permittivity and permeability in the microwave frequency band [1], [2]. His structures as well as the other resonant types are basically fabricated with patterned printed circuit boards (PCB) or metal objects aligned periodically on a plate. Considering the difficulty to align the PCBs in the three dimensional structure, these metamaterials should naturally be anisotropic.

This article will analyze propagating modes in an anisotropic slab with negative permeability or permittivity tensor components. Since there is a unique surface mode along the boundary of positive and negative materials where the field decays exponentially to both directions perpendicular to it [3], we call the mode that varies sinusoidally inside the slab as a volume mode for the sake of clear discrimination. We will find the correspondence of the volume and surface modes to the sign of each component of the tensor material constant.

By the inspection with the quasi-static approach, the obtained electromagnetic wave suggests the possibility of magnetostatic wave for the case of negative permeability and electrostatic wave for negative permittivity, respectively.

\section{Volume Mode Of Single-Negative Slab WITH Tensor Material Parameters}

\section{A. Basic Structure}

As shown in Fig. 1, we analyze the TE mode in finite-thickness slab with tensor permittivity $\hat{\varepsilon}$ and permeability $\hat{\mu}$ whose components are allowed to be negative and the thickness is $T$. The coordinate system used in the analysis is also shown in the same figure. It is assumed that the tensor permittivity and permeability of slab region (region II) are linear and simultaneously diagonalizable,

$$
\hat{\varepsilon}=\left(\begin{array}{ccc}
\varepsilon_{x} & 0 & 0 \\
0 & \varepsilon_{y} & 0 \\
0 & 0 & \varepsilon_{z}
\end{array}\right), \quad \hat{\mu}=\left(\begin{array}{ccc}
\mu_{x} & 0 & 0 \\
0 & \mu_{y} & 0 \\
0 & 0 & \mu_{z}
\end{array}\right) .
$$

The upper and lower regions of the slab are the free-space with the material parameters $\varepsilon_{0}$ and $\mu_{0}$ (regions I and III).

\section{B. Field Expression and Eigenvalue Equation}

Starting from the source-free Maxwell's equation, the EM field profiles for TE volume modes can be expressed in each region as follows,

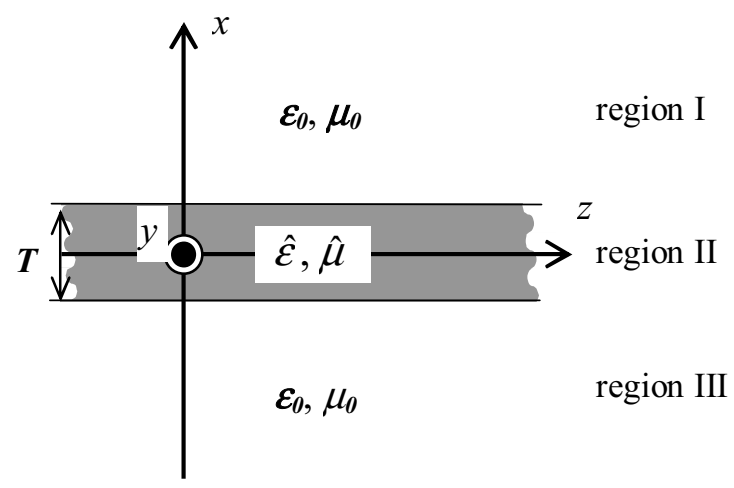

Fig.1 Finite-thickness slab with anisotropic permittivity and/or permeability. 


$$
\begin{aligned}
& \left\{\begin{array}{l}
H_{x}=-\frac{j \beta C}{\delta_{0}} e^{-\delta_{0} x} \\
H_{z}=C e^{-\delta_{0} x} \quad\left(x \geq \frac{T}{2}\right), \\
E_{y}=\frac{j \omega \mu_{0} C}{\delta_{0}} e^{-\delta_{0} x}
\end{array}\right. \\
& \left\{\begin{array}{l}
H_{x}=-\frac{j \mu_{z} \beta}{\mu_{x} \delta_{1}}\left(-A \sin \delta_{1} x+B \cos \delta_{1} x\right) \\
H_{z}=A \cos \delta_{1} x+B \sin \delta_{1} x \\
E_{y}=\frac{j \omega \mu_{z}}{\delta_{1}}\left(-A \sin \delta_{1} x+B \cos \delta_{1} x\right)
\end{array} \quad\left(|x| \leq \frac{T}{2}\right),\right. \\
& \left\{\begin{array}{l}
H_{x}=\frac{j \beta D}{\delta_{0}} e^{\delta_{0} x} \\
H_{z}=D e^{\delta_{0} x} \\
E_{y}=-\frac{j \omega \mu_{0} D}{\delta_{0}} e^{\delta_{0} x}
\end{array} \quad\left(x \leq-\frac{T}{2}\right),\right.
\end{aligned}
$$

where $A, B, C$ and $D$ are the arbitrary constants and

$$
\delta_{0}=\sqrt{\beta^{2}-\omega^{2} \varepsilon_{0} \mu_{0}}, \quad \delta_{1}=\sqrt{\left(\mu_{z} / \mu_{x}\right)\left(\omega^{2} \varepsilon_{y} \mu_{x}-\beta^{2}\right)} .
$$

Applying the boundary conditions at $x= \pm T / 2$ surfaces, the following eigenvalue equation for $\mathrm{TE}$ mode can be obtained.

$$
\left(\mu_{0}^{2} \delta_{1}^{2}-\mu_{z}^{2} \delta_{0}^{2}\right) \sin \delta_{1} T=2 \mu_{0} \mu_{z} \delta_{0} \delta_{1} \cos \delta_{1} T .
$$

To check the validity of eigenvalue equation (3), we derive that of TE surface mode presented in Ref. [3] from Eq.(3). In order to express the finite-thickness single-negative sheet with negative scalar permeability and positive scalar permittivity, we set the components of the permittivity and the permeability tensors as $\varepsilon_{x}=\mathcal{E}_{y}=\varepsilon_{z}=\varepsilon_{1}$ and $\mu_{x}=\mu_{z}=-\mu_{1}$ where $\varepsilon_{1}$ and $\mu_{1}$ are the positive real quantities. When the components of material parameters are set as above, the phase constant $\delta_{1}$ becomes imaginary, being expressed as $\delta_{1}=j \sqrt{\omega^{2} \varepsilon_{1} \mu_{1}+\beta^{2}}=j \delta_{1}$ ' with real quantity $\delta_{l}$ '. By substituting $\delta_{1}$ ' into Eq.(3), we can get the same eigenvalue equation for TE surface mode appeared in Eq.(10) of Ref. [3]. Here, note that the $y$-component of the permeability tensor is not included in the above analysis. This means that TE surface and volume modes can propagate along the slab waveguide, regardless of the $y$-component of permeability tensor. In Table 1, we summarize the relationship between the sign of permeability tensor components and the modes propagating to the $z$-direction in the slab extending to $y$-and $z$-directions as shown in Fig.1.

For a slab with negative permittivity tensor, on the other hand, TM mode dispersion relation and field distributions will be easily obtained with proper interchange of the electric and magnetic field components due to the duality of the electric and magnetic fields in the similar manner as the TE modes.

\begin{tabular}{|c|c|c|c|c|c|}
\hline & $\mu_{\mathrm{x}}$ & $\mu_{\mathrm{y}}$ & $\mu_{\mathrm{z}}$ & $\delta_{1}$ & \\
\hline a) & + & \multirow{4}{*}{ \pm} & \multirow{2}{*}{-} & \multirow{2}{*}{ Imag. } & \multirow{2}{*}{ TE surface mode } \\
\hline b) & - & & & & \\
\hline c) & - & & \multirow[b]{2}{*}{+} & \multirow[b]{2}{*}{ Real } & TE volume mode \\
\hline d) & + & & & & $\begin{array}{l}\text { Conventional } \\
\text { TE volume mode }\end{array}$ \\
\hline
\end{tabular}

TABLE 1

Existence of TE modes in slab shown in Fig.1 with possible sign combinations of permeability tensor. (All components of permittivity tensor are assumed as positive quantities.)

\section{Volume Modes in Single-Negative Slab With Negative Permeability Tensor}

\section{A. Dispersion Characteristics}

The metamaterial fabricated by stacking the substrate with periodically embedded Swiss-Roll capacitors (SRCs) as shown in Fig. 2 would exhibit a negative and dispersive permeability, but positive and non-dispersive permittivity in the $x$-direction. We set the $x$-components of permeability and permittivity tensors as $\mu_{\mathrm{x}}=\mu_{\mathrm{r}}(\omega) \mu_{0}$ and $\varepsilon_{\mathrm{x}}=\varepsilon_{\mathrm{r}} \varepsilon_{0}$ as was expressed in Ref.[1] with the resistance of SRCs $\sigma_{1}=0.0$,

$$
\mu_{r}(\omega)=1-\frac{\pi r^{2} / a^{2}}{1-\left\{d c_{0}^{2} / 2 \pi^{2} \omega^{2}(N-1) r^{3}\right\}}, \varepsilon_{r}=\left(1-\frac{\pi r^{2}}{a^{2}}\right)^{-1}
$$

where $c_{0}$ is the velocity of light and the other parameters of

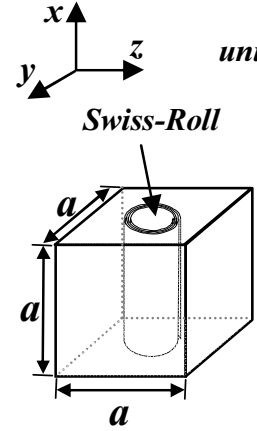

(a) Unit cell

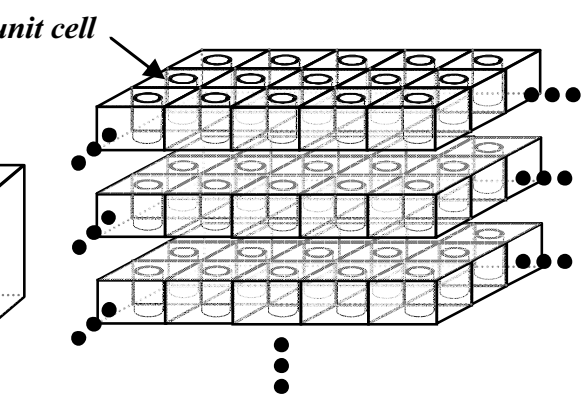

(b) Stacking sequence of unit sheets

Fig.2 Structure of metamaterial with negative permeability in $x$-direction.

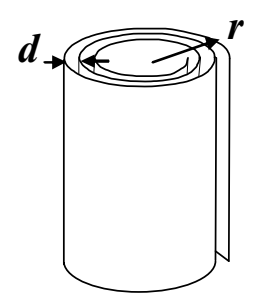

N turns

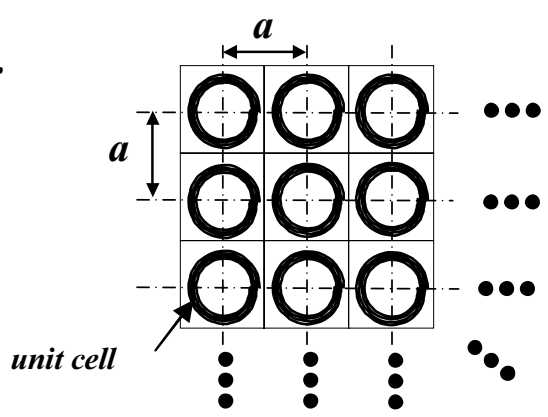

(b) Stacking and arranging sequence $r=2.0 \times 10^{-4} \mathrm{~m}, a=5.0 \times 10^{-4} \mathrm{~m}, d=1.0 \times 10^{-5} \mathrm{~m}, \mathrm{~N}=3$

Fig.3 Dimensions and definition of distances for SRCs [1]. 
SRCs are shown in Fig.3. The $\mu_{\mathrm{r}}(\omega)$ takes negative value over the frequency range from the singular point at $8.50 \mathrm{GHz}$ to $12.04 \mathrm{GHz}$ and is smaller than -1.0 below $9.81 \mathrm{GHz}$. In our analysis, the remaining components of material tensors and the slab thickness are assumed as $\varepsilon_{x}=\varepsilon_{y}$ $=\varepsilon_{z}=2.01 \varepsilon_{0}, \mu_{v}=\mu_{z}=\mu_{0}$, and $T=5.0 \mathrm{~mm}$.

Substituting Eq.(4) into (3), we show the dispersion characteristics of TE volume modes with thick-solid lines in Fig.4. As the operating frequency increases, the propagation constants of $\mathrm{TE}_{2}$ volume mode and the other higher order volume modes go down toward the cutoff frequency $9.81 \mathrm{GHz}$ where $\mu_{\mathrm{x}}$ is close to $-\mu_{0}$. These natures tell us that these TE modes are backward waves having the same property as the modes in double-negative material. It should be noted, however, that the different dispersion characteristics is observed for $\mathrm{TE}_{1}$ volume mode. Two eigenvalues for $\mathrm{TE}_{1}$ volume mode at the same operating frequency can be obtained and their tendencies of the dispersion curves can be tentatively classified into two branches (i) and (ii), which are also indicated in Fig.4. Branch (i) behaves as a backward wave in the same way as other higher modes, while branch (ii) is forward. The electromagnetic field profiles $H_{z}$ and $E_{y}$ for $\mathrm{TE}_{1}$ volume mode in each branch and those of $\mathrm{TE}_{2}$ volume mode observed at $8.8 \mathrm{GHz}$ are presented in Fig.5, respectively. From these results, we know that the fields vary sinusoidally inside the slab and the both field profiles for branch (i) and (ii) of $\mathrm{TE}_{1}$ volume mode are quite similar to each other.

These facts verify that all of obtained TE modes are the volume modes, but only $\mathrm{TE}_{1}$ volume mode has special feature and its nature differs from that of other higher order modes.

\section{B. Time-Averaged Electric and Magnetic Energy}

In order to investigate the nature of these modes in more detail, we estimate the time-averaged energy stored in the EM field. The electric and magnetic stored energy for unit length of the slab waveguide is given, respectively, by the following integral expressions [4],

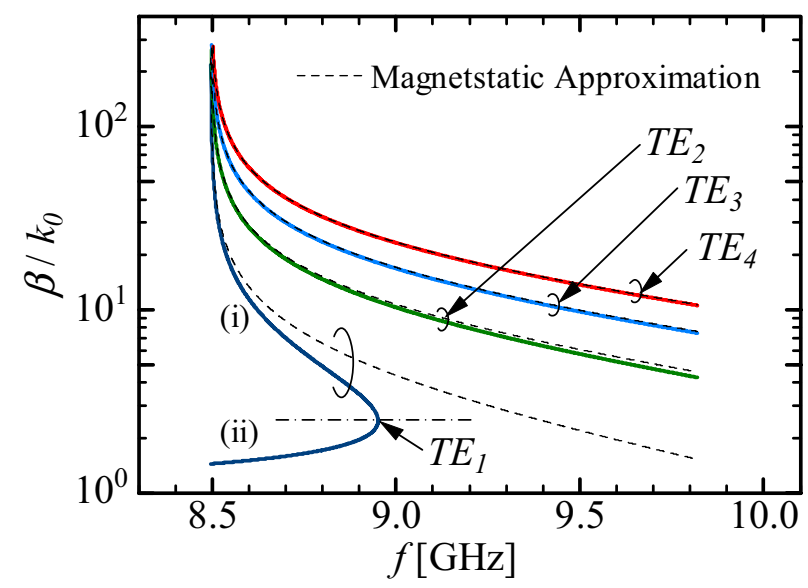

Fig. 4 Dispersion characteristics for TE volume modes in the single-negative slab with tensor permeability.

$$
\begin{aligned}
& W_{e}=\frac{\varepsilon_{0}}{4} \int_{-\infty}^{\infty} \mathbf{E} \cdot \mathbf{E}^{*} \frac{\partial\left\{\omega \varepsilon_{r}(\omega)\right\}}{\partial \omega} d x, \\
& W_{m}=\frac{\mu_{0}}{4} \int_{-\infty}^{\infty} \mathbf{H} \cdot \mathbf{H}^{*} \frac{\partial\left\{\omega \mu_{r}(\omega)\right\}}{\partial \omega} d x
\end{aligned}
$$

where $\varepsilon_{r}(\omega)$ and $\mu_{r}(\omega)$ are the relative permittivity and permeability with dispersion, respectively. In our analysis, it is assumed that the permittivity of the material has no dispersion but the permeability in the $x$-direction is dispersive. Substituting the electromagnetic fields Eq.(2) and the permeability/permittivity Eq.(4) into Eq.(5), we can obtain the energy stored in EM fields of single-negative finite-thickness slab with the negative permeability tensor.

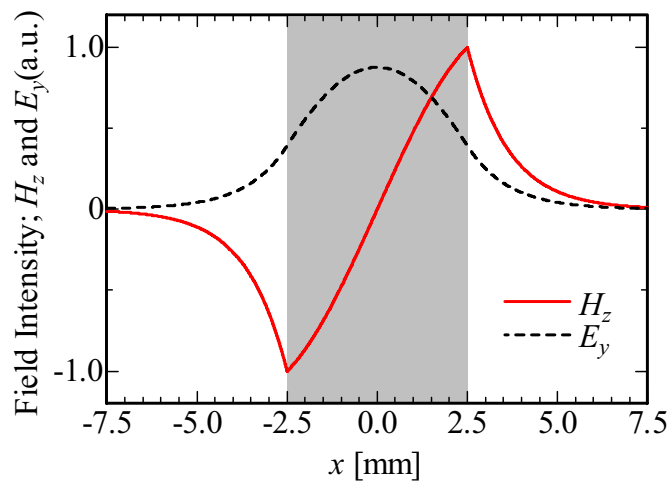

(a) $\mathrm{TE}_{1}$ mode on branch (i)

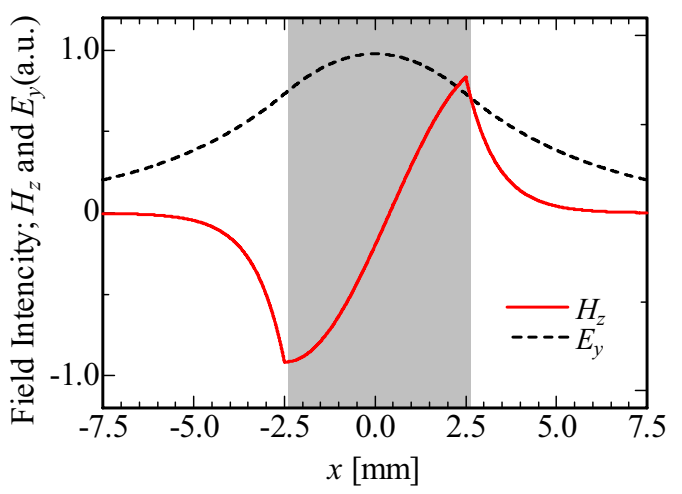

(b) $\mathrm{TE}_{1}$ mode on branch (ii)

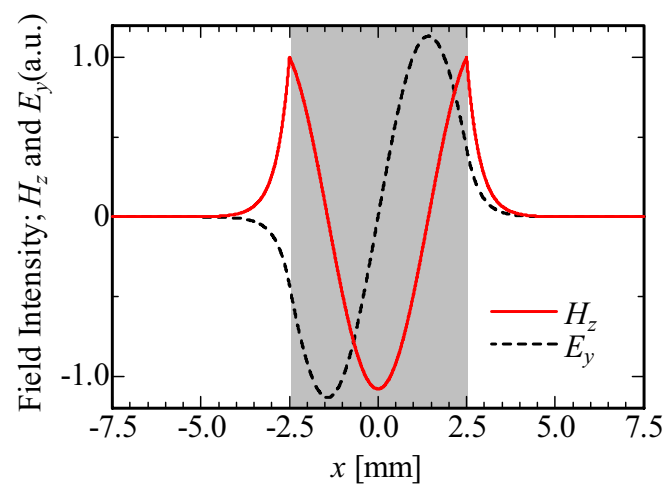

(c) $\mathrm{TE}_{2}$ mode

Fig. 5 Electromagnetic field profiles for TE volume modes with $T=5.0 \mathrm{~mm}$ and $f=8.8 \mathrm{GHz}$. 
Figure 6 presents the time-averaged magnetic energy as well as the ratio of the electric and magnetic energy for the $\mathrm{TE}_{1}$ and $\mathrm{TE}_{2}$ modes. The same tendencies as $\mathrm{TE}_{2}$ mode can be obtained for the modes with higher than the second order. From the result of Fig.6(a), the magnetic energy is larger than electric energy, but their ratio for branch (i) becomes unity or less around $8.7 \mathrm{GHz}$. This means that the electric and magnetic energy of $\mathrm{TE}_{1}$ mode are equalized over some frequency region, which might suggest that only a part of $\mathrm{TE}_{1}$ mode is magnetostatic wave. On the contrary, the result of Fig.6(b) tells us that the energy stored in the magnetic field of $\mathrm{TE}_{2}$ and higher order modes are extremely larger than the electric counterpart, which suggests that those $\mathrm{TE}_{2}$ and the other higher order waves could be magnetostatic waves.

\section{Magnetostatic Approximation}

The magnetostatic approximation

$$
\nabla \times \mathbf{H}=\mathbf{0}, \quad \nabla \cdot \hat{\mu} \mathbf{H}=0
$$

gives the following wave equation for the scalar potential $\psi$ in the slab defined by Eq.(4)

$$
\mu_{x} \frac{\partial^{2} \Psi}{\partial x^{2}}-\beta^{2} \mu_{0} \Psi=0
$$

The dispersion equations are easily obtained

$$
\begin{aligned}
& \cot \frac{\beta T}{2 \sqrt{\mu_{a}}}=-\sqrt{\mu_{a}} \quad \text { even mode, } \\
& \tan \frac{\beta T}{2 \sqrt{\mu_{a}}}=\sqrt{\mu_{a}} \quad \text { odd mode, }
\end{aligned}
$$

by applying the boundary conditions for the potential at $x= \pm T / 2$ in Fig. 1 , where we set as $\mu_{\mathrm{x}}=-\mu_{\mathrm{a}} \mu_{0}$ and $\mu_{\mathrm{a}}=\left|\mu_{\mathrm{r}}(\omega)\right|$. The dispersion curves for TE modes numerically obtained from Eq.(8) are also shown in Fig.4 with thin-dashed lines. Except $\mathrm{TE}_{1}$ mode, the dispersion curves obtained from Eq.(8) agree well with those obtained from the eigenvalue equation (3). On the other hand, both branches for the $\mathrm{TE}_{1}$ mode disagree with the MSW approximation and their difference becomes larger at the higher frequency range. These facts tell us that the $\mathrm{TE}_{2}$ and other higher order modes presented in this article are magnetostatic wave, but $\mathrm{TE}_{1}$ mode is not.

\section{Conclusions}

The eigenvalue equation and field expressions of volume modes for the finite-thickness slab with single-negative tensor and dispersive material parameters were demonstrated. We have revealed that the nature of the existing modes in the single-negative slab, whether surface mode or volume mode, depends on the sign combination of the tensor components of material parameters.

It is presented that the TE volume mode obtained in this paper is the magnetostatic wave by magnetostatic approximation analysis. Thus, it has been brought to light

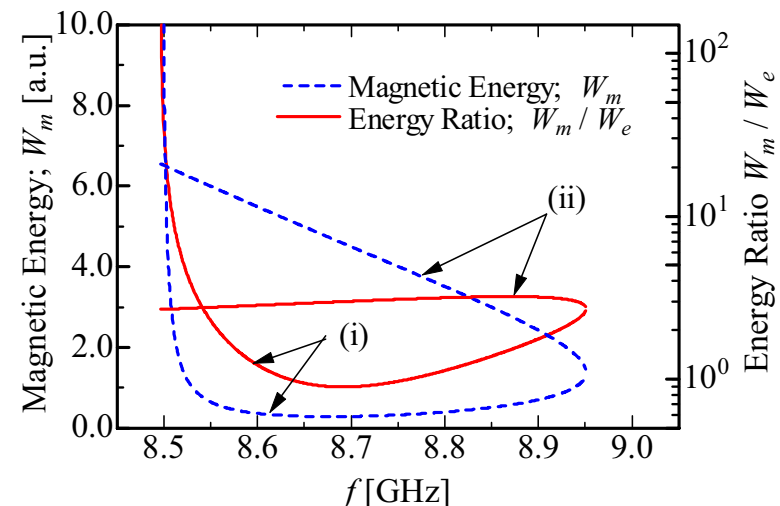

(a) $\mathrm{TE}_{1}$ mode

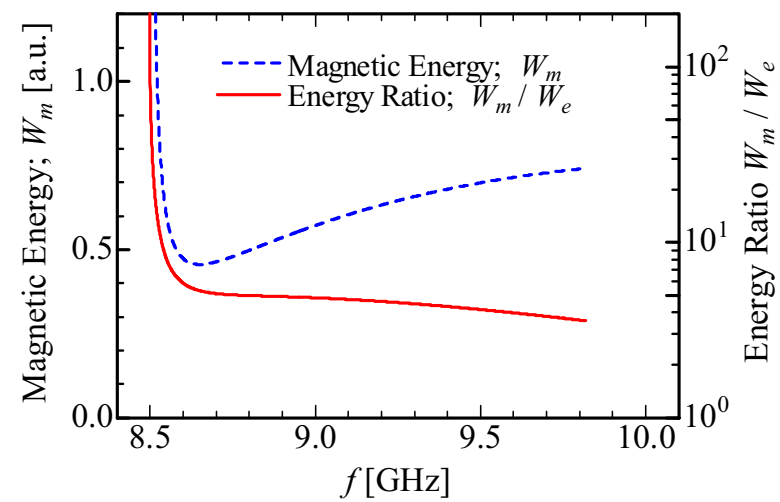

(b) $\mathrm{TE}_{2}$ mode

Fig. 6 Time-average electric and magnetic energy stored in the TE modes.

that the magnetostatic wave could exist in nonmagnetic materials. The similar analysis to TM modes in a slab with negative permittivity will show the existence of electrostatic waves. This fact would bring us the possibility of novel microwave and millimeter-wave applications with single-negative metamaterial.

\section{ACKNOWLEDGEMENT}

This work was partly supported by the MEXT, Japan, Grant-in-Aid for Scientific Research (C) 18560335.

\section{REFERENCES}

[1] J. B. Pendry, A. J. Holden, D. J. Robbins and W. J. Stewart, "Magnetism from Conductors and Enhanced Nonlinear Phenomena," IEEE Trans. on Microwave Theory \& Tech., vol.47, no.11, pp.2075-2081, Nov. 1999.

[2] J. B. Pendry, A. J. Holden, W. J. Stewart and I. Youngs, "Extremely Low Frequency Plasmons in Metallic Mesostructure," Phys. Rev. Lett., vol.76, pp.4773-4776, 1996.

[3] M. Hotta, M. Hano and I. Awai, "Surface Waves along a Boundary of Single Negative Material," IEICE Trans. on Electron., vol.E88-C, no.2, pp.275-278, 2005.

[4] Robert E. Collin, Foundations for Microwave Engineering, 2nd Ed., McGraw-Hill, 1992, Chap.2. 